\title{
DIFERENCIACION SOCIO RESIDENCIAL, UNA APLICACIÓN PRÁCTICA AL ESPACIO URBANO DEL GRAN RESISTENCIA
}

\author{
DIFFERENTIATION RESIDENTIAL PARTNER, A PRACTICAL APPLICATION TO THE \\ URBAN SPACE OF THE GREAT RESISTANCE
}

Mgtr. Vilma Lilian Falcón

Prof. Adjunta del Departamento de Geografía

Facultad de Humanidades - UNNE

\section{RESUMEN}

El análisis de la diferenciación socio espacial es una aproximación básica para el estudio del espacio geográfico. Si bien, como lo señalan Buzai y Baxendale (2006:335) estos estudios se dieron en el ámbito de la sociología urbana, con una aplicación metodológica para medir la concentración y segregación espacial en ámbitos intraurbanos y de determinados segmentos poblacionales especialmente migrantes, en la actualidad las metodologías y técnicas que intentan medir estos fenómenos han experimentado una revalorización y ampliación en el uso de estas líneas de análisis.

En este sentido, el trabajo que se realiza consiste básicamente en analizar, a la luz de las aportaciones teóricas, cómo se manifiestan los elementos más significativos de diferenciación socioespacial en la ciudad de Resistencia, capital de la provincia del Chaco, a la vez que se ensayan algunas aplicaciones de cálculo estadístico con los indicadores socioeconómicos.

\section{PALABRAS CLAVES}

Diferenciación Socioespacial; Segregación espacial; Modelos teóricos urbanos; Resistencia; Chaco.

\section{SUMARY}

The analysis of the differentiation space partner is a basic approach for the study of the geographic space. Although, as they indicate Buzai and Baxendale to it (2006: 335) these studies occurred in the scope of urban sociology, with a methodologic application to measure the concentration and space segregation in intraurban scopes and of certain specially migrantes population segments, at the present time the methodologies and techniques that try to measure these phenomena have experienced a revaluation and extension in the use of these lines of analysis.

In this sense, the work that is made consists basically in analyzing, to the light of the theoretical contributions, how the most significant elements of socioespacial differentiation in the city of Resistance are pronounced, capital of the province of the Chaco, simultaneously that try some applications of statistical calculation with the socioeconomic indicators.

\section{KEY WORDS}

Socioespacial differentiation; space Segregation; Urban Theoretical Models; Resistencia; Chaco

\section{SUMARIO DEL ARTÍCULO}

1- Introducción

2- Unidades espaciales y variables

3- El espacio de estudio y las particularidades observadas

4- Distribución espacial de las variables consideradas en este estudio

5- Hacia la búsqueda de un modelo

\section{1.- Introducción}

El objetivo del presente trabajo es dar cumplimiento a la propuesta de evaluación planteada por el curso de Postgrado: Diferenciación socio-residencial. Definiciones, interpretaciones y métodos de medición ${ }^{(1)}$. En este sentido, el trabajo que se realiza consiste básicamente en analizar, a la luz de las aportaciones teóricas, cómo se manifiestan los elementos más significativos de diferenciación socioespacial en la ciudad de Resistencia, capital de la provincia del Chaco. Basándonos en algunos indicadores específicos provenientes del Censo Nacional de Población y Viviendas del año 2001, se determinarán las particularidades de la distribución espacial teniendo en cuenta aspectos demográficos y sociales en el Gran Resistencia. 
Por otra parte, intentaremos enmarcar la realidad estadística de Resistencia y el resultado de las distribuciones que resultan de los mapas, en el marco más amplio de los modelos teóricos a través de los cuales se analiza la estructura espacial de las ciudades.

\section{2.-Unidades espaciales y variables}

Para la realización de este ejercicio de aplicación se ha considerado el nivel de radio censal como unidad espacial, siendo la principal fuente de información la Base Usuario REDATAM del Censo Nacional de Población y Vivienda 2001. En cuanto a la cartografía hemos utilizado la base gráfica del Gran Resistencia que contiene 368 unidades en total. La información contenida en dichas unidades espaciales corresponden a 12 variables socio demográficas, por lo tanto nuestra matriz de datos originales queda conformada por 368 radios y 12 variables.

\section{3.-El espacio de estudio y las particularidades observadas}

La ciudad de Resistencia, capital de la provincia del Chaco conforma, junto a otros tres municipios (Fontana, Barranqueras y Vilelas) el conglomerado llamado Gran Resistencia, tal como puede observarse en el mapa 1. De acuerdo a los datos del Censo del año 2001, este conglomerado contaba con aproximadamente 360.000 habitantes.

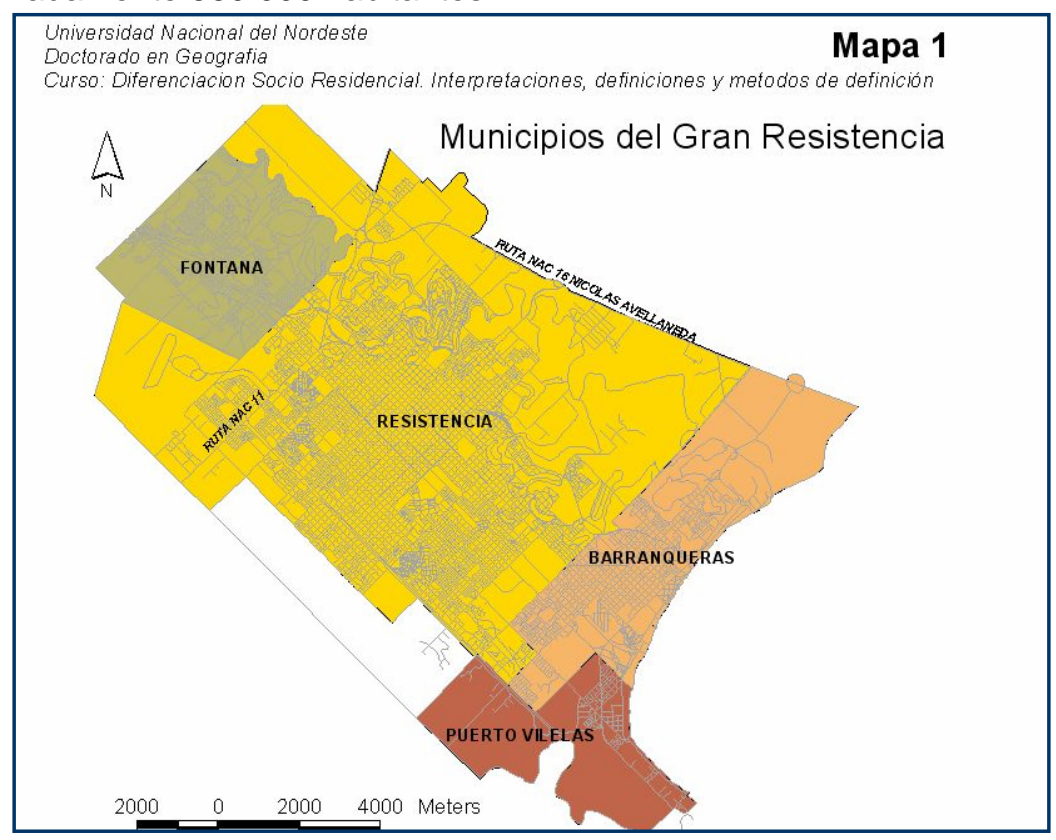

Podríamos decir que lo señalado por Borsford, A.(2003) en su estudio de los modelos de desarrollo y la dinámica de la ciudad latinoamericana ${ }^{(2)}$, bien puede aplicarse al dinamismo que siguió la ciudad de Resistencia, en el sentido que, desde su nacimiento en 1878 hasta la actualidad, esta ciudad ha seguido un esquema de configuración como resultado de distintas fases de urbanización por la que fue atravesando, es decir, en forma similar a las señaladas por el autor, las que responden a la época colonial, la primera fase de urbanización influida fuertemente por la inmigración europea, la segunda fase de urbanización marcada por el éxodo rural y la migración interna y la ciudad contemporánea.

Si bien en este trabajo no nos ocuparemos del análisis de cada una de las fases que puedan explicar el desarrollo urbano de nuestra ciudad, nos interesa destacar que en los últimos años el Gran Resistencia sufrió un proceso de expansión urbana muy importante. Lo que podemos afirmar es que en ese proceso de expansión, esta ciudad no escapó, como también lo afirma Borsdford, a la "norma urbana de la segregación", plasmada en una clara diferenciación socio-espacial que se manifiesta en la estructura urbana y que resulta observable a simple vista para quienes la transitamos cotidianamente.

Vale decir que consideramos, desde un inicio, que el Gran Resistencia presenta rasgos de diferenciación socio residencial cuya plasmación espacial puede ser observada en forma directa.

Ahora bien, de lo que cotidianamente observamos: ¿Qué elementos o aspectos de la población están reflejando esa diferenciación que se plasma en el espacio urbano? ¿hay, en la

Publicado en formato digital: Mgtr. Vilma Lilian Falcón. DIFERENCIACION SOCIO RESIDENCIAL, UNA APLICACIÓN PRÁCTICA AL ESPACIO URBANO DEL GRAN RESISTENCIA. Revista Geográfica Digital. IGUNNE. Facultad de Humanidades. UNNE. Año 8. No 15. Enero - Junio 2011. ISSN 1668-5180 Resistencia, Chaco. En: http://hum.unne.edu.ar/revistas/geoweb/default.htm 
combinación de elementos sociales y demográficos, algunos que inciden con mayor peso que otros en esa diferenciación espacial? ¿pueden asociarse a la configuración espacial de esta ciudad algunos de los modelos teóricos que diversos autores han propuesto para el estudio del crecimiento de las ciudades? Si esto es así, ¿qué modelo se ajusta más a la realidad del Gran Resistencia?

El análisis que realizaremos está orientado a dar respuesta a estos interrogantes planteados, dado que la afirmación que hemos hecho esta basada solo en nuestra percepción de la realidad; por lo tanto debe ser -parafraseando a Rodríguez Vignoli, J. (2001:10), "motivo de una indagación empírica".

\section{4.- Distribución espacial de las variables consideradas en este estudio}

Señala Sabatini (2003) ${ }^{(3)}$ que, en términos generales, la segregación residencial corresponde a la aglomeración -en el espacio- de familias de una misma condición social, más allá de cómo se definan las diferencias sociales. Expresa el autor que la segregación puede ser según condición étnica, origen migratorio, etaria o socioeconómica, entre otras. En nuestro caso trabajaremos con los datos de población por grupos etarios y condiciones socioeconómicas. Esto es válido ya que -como lo mencionáramos en los puntos anteriores-, más allá de lo que se puede observar en forma directa, el comportamiento de algunas de las variables en el espacio urbano solo puede ser advertido a través de la representación cartográfica.

Señalábamos en el punto 1.1, que los datos correspondientes a las 12 variables provienen de la información que provee la Base Usuario REDATAM del Censo Nacional de Población y Vivienda 2001. A los efectos de su mejor interpretación agruparemos dichos datos según respondan a las características de variables referidas a los grupos etarios de la población y a las condiciones de educación y ocupación.

En todos los casos adoptamos el criterio de mantener el intervalo de los grupos según los quiebres o cortes naturales con los que agrupa el software empleado (ArcGis3.2)

\section{1.- Distribución espacial de la población según grupos de edad}

El mapa 2 nos muestra la distribución porcentual de población menor a 14 años de edad; en él, se observa claramente que las mayores proporciones corresponden a los radios periféricos de la ciudad, destacándose el área céntrica de la ciudad de Resistencia por la baja presencia de población joven.

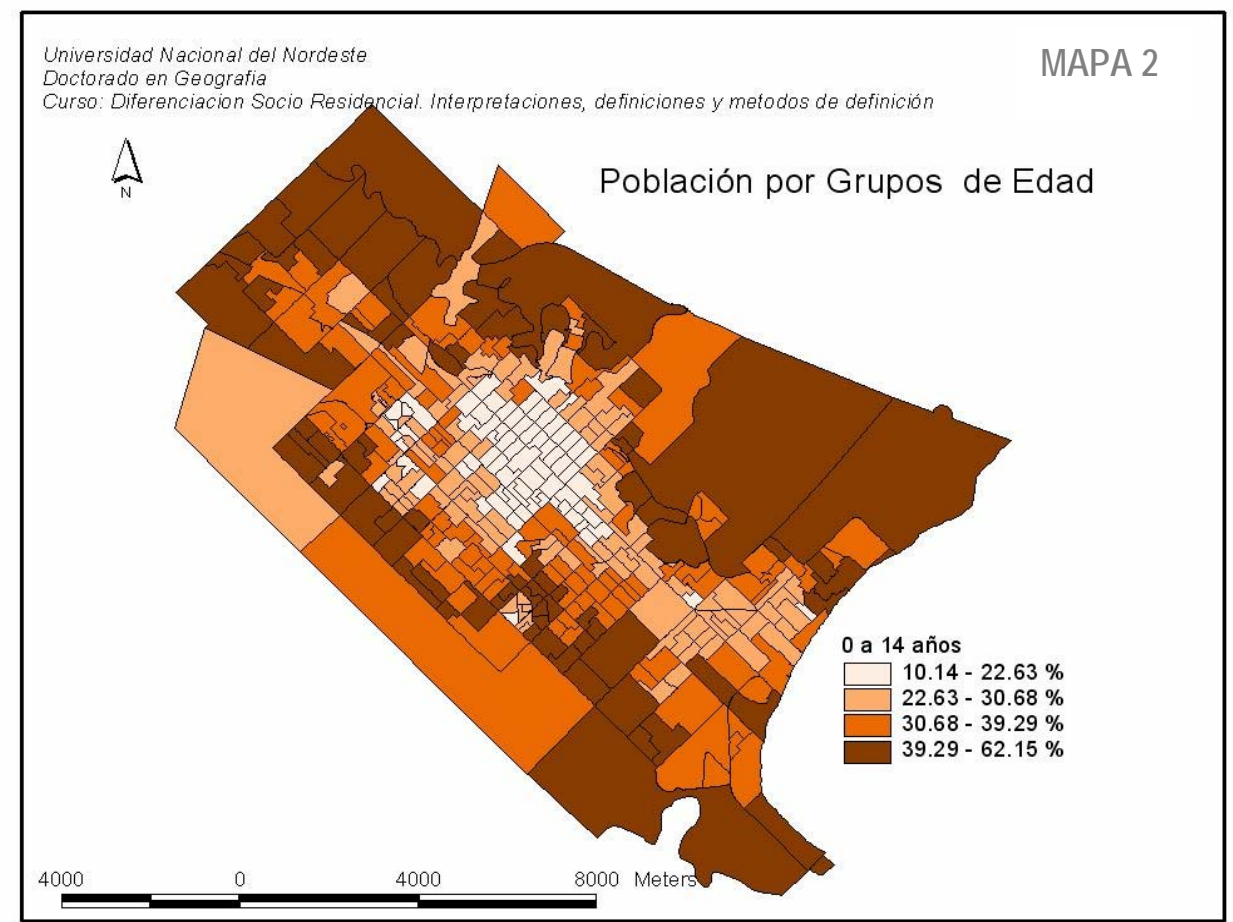

Publicado en formato digital: Mgtr. Vilma Lilian Falcón. DIFERENCIACION SOCIO RESIDENCIAL, UNA APLICACIÓN PRÁCTICA AL ESPACIO URBANO DEL GRAN RESISTENCIA. Revista Geográfica Digital. IGUNNE. Facultad de Humanidades. UNNE. Año 8. No 15. Enero - Junio 2011. ISSN 1668-5180 Resistencia, Chaco. En: http://hum.unne.edu.ar/revistas/geoweb/default.htm 
La distribución de los porcentuales es totalmente opuesta, como se verá en el Mapa 3, al grupo de población adulta, es decir la que representa a la población mayor a 65 años.

La forma que presenta la distribución del grupo mayor a 65 años en el espacio urbano, permite advertir claramente un núcleo central con alta concentración de población adulta que disminuye hacia la periferia en forma de "anillos concéntricos", aunque se delinean claramente cuatro ejes de mayores proporciones siguiendo las principales avenidas del conglomerado; esta forma también se traslada a un núcleo que comienza a definirse -hacia el año 2001- en la ciudad portuaria de Barranqueras

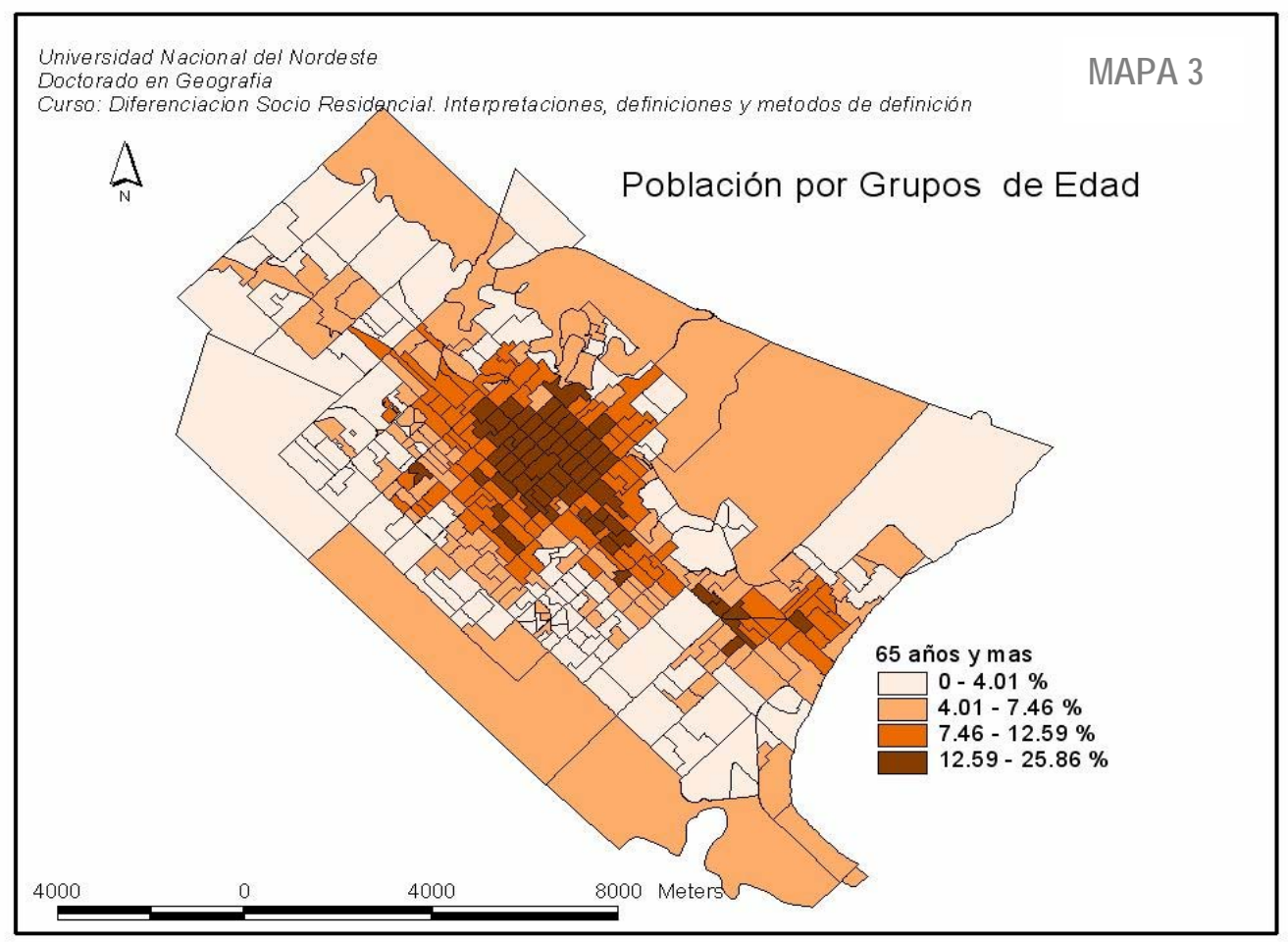

Este hecho puede deberse a que la población asentada desde hace mucho tiempo en espacios céntricos y aledaños permanece en esos lugares y las nuevas generaciones, compuestas por jóvenes que forman su propia familia, se trasladan hacia la periferia ya sea porque es allí donde se encuentran terrenos disponibles para construir o bien porque han sido beneficiados por los planes habitacionales que provee el Estado, los que, generalmente construyen nuevos barrios en zonas periféricas o fuera del macrocentro dada la necesidad del espacio físico requerido.

Si bien, a primera impresión, esta localización de población adulta en el centro podría parecer beneficiosa para este grupo, dada la proximidad a los principales servicios bancarios, financieros, comerciales entre otros, hay que considerar otros elementos que pueden actuar en forma negativa, como por ejemplo el compartir con pobladores circunstanciales un espacio urbano altamente densificado, especialmente en horarios comerciales, lo que lleva a este grupo de población a la necesidad de ajustarse al ritmo particular de estos lugares; ello genera la consiguiente dificultad para trasladarse hacia los lugares de trámites cotidianos; si a ello sumamos un diseño de ciudad centro que no está pensada precisamente para una población que ya no mantiene una habilidad motriz para desplazarse en veredas, calles, cruces de calles, etc. nos encontramos apenas en el inicio de un largo listado de eventos negativos o desfavorables que podrían dar cuenta de que en cierta manera esta población adulta mayor, concentrada en los espacios centrales de la ciudad, presenta cierto rasgo de segregación.

En lo que respecta a estas dos primeras variables podemos sintetizar que:

1) existe un núcleo conformado por el casco céntrico de la ciudad que se distingue, algunas veces muy claramente y otras en forma más difusa, del resto del espacio estudiado. Esto es observable tanto por la presencia o por la ausencia en los porcentuales de los datos 
representados. La concentración de población joven va adquiriendo mayores proporciones desde el centro hacia la periferia.

Por su parte, la distribución del grupo mayor a 65 años en el espacio urbano, conforma un núcleo central con alta concentración de población adulta que disminuye hacia la periferia,

2) De acuerdo a los modelos de ciudad estudiados, la forma resultante puede asociarse primero, en forma más general al modelo de anillos concéntricos propuesto por Burguess ${ }^{(4)}$, solamente si consideramos que desde el centro o CBD, los elementos representados se van diferenciando, por su mayor o menor presencia, hacia la periferia y por lo tanto la distancia a dicho CBD puede considerarse como un factor de diferenciación. Pero, si consideramos la forma de la ciudad de Resistencia y la disposición de los ejes principales de comunicación, podemos señalar que el modelo que se ajusta con mas precisión es el modelo sectorial de Hoytt (1939) ${ }^{(5)}$; en este caso determinado por la importancia que adquieren las principales avenidas que comunican al centro urbano con la periferia de la ciudad.

\section{2.- Distribución espacial de la población según indicadores Socioeconómicos}

En primer lugar, describiremos las condiciones de actividad de la población y su posible correspondencia con el nivel educativo de la misma. Para ello, se representaron espacialmente los siguientes indicadores: población que cuenta con educación universitaria finalizada, población que asiste a establecimientos privados, población ocupada no calificada y, finalmente, la proporción de población ocupada a la que no le aportan ni hacen descuentos.

En lo que respecta a la distribución espacial de los indicadores referidos a la educación de la población (Mapas 4 y 5), debemos decir que el mapa que representa la proporción de población universitaria es el que refleja una mayor concentración espacial en la ciudad, correspondiéndole las mayores proporciones a los radios de área centrales de la ciudad de Resistencia y a otros que se presentan en forma de "islas". Por su parte, la configuración que adopta el mapa que representa la proporción de población que asiste a establecimientos educativos privados permite advertir una configuración similar, aunque más extendida en el espacio urbano, abarcando además los radios próximos a las principales avenidas en las ciudades de Resistencia y Barranqueras.

Los Mapas 6 y 7 muestran dos particularidades de la población ocupada; por un lado se muestra cómo las personas ocupadas que no reciben aportes de ningún tipo se distribuyen en mayores proporciones en los radios más periféricos de la ciudad, dejando algunos intersticios de menor valor en dichos espacios; por otra parte, el área del centro urbano muestra una menor cantidad de población con esta característica. Si hiciéramos una superposición de los dos mapas podríamos advertir que la mayor correspondencia se da en los radios céntricos con bajas proporciones de población no calificadas y ocupados sin aportes. En general, los radios periféricos son los que presentan las situaciones más desfavorables en el análisis de esta variable.

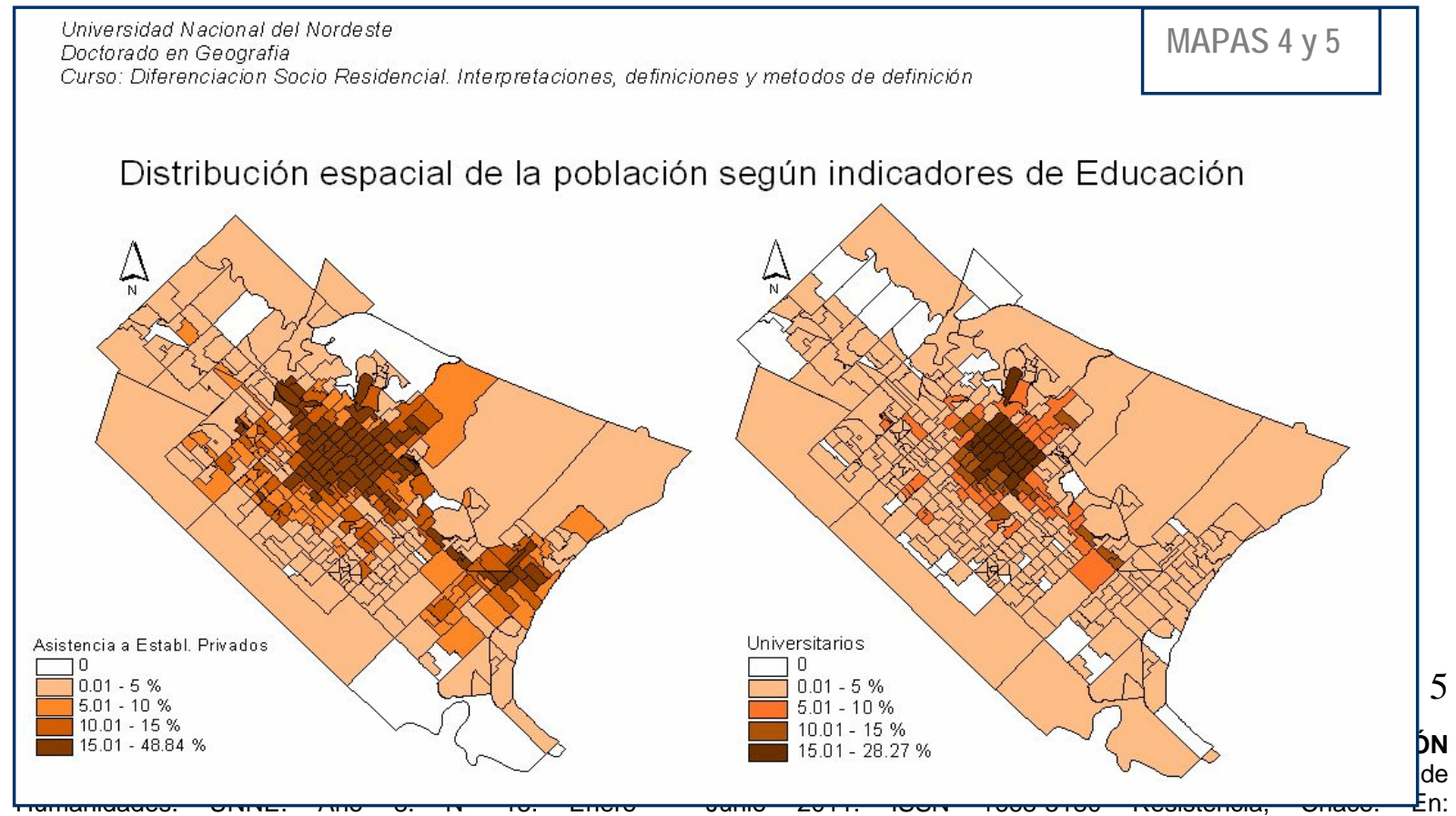

http://hum.unne.edu.ar/revistas/geoweb/default.htm 


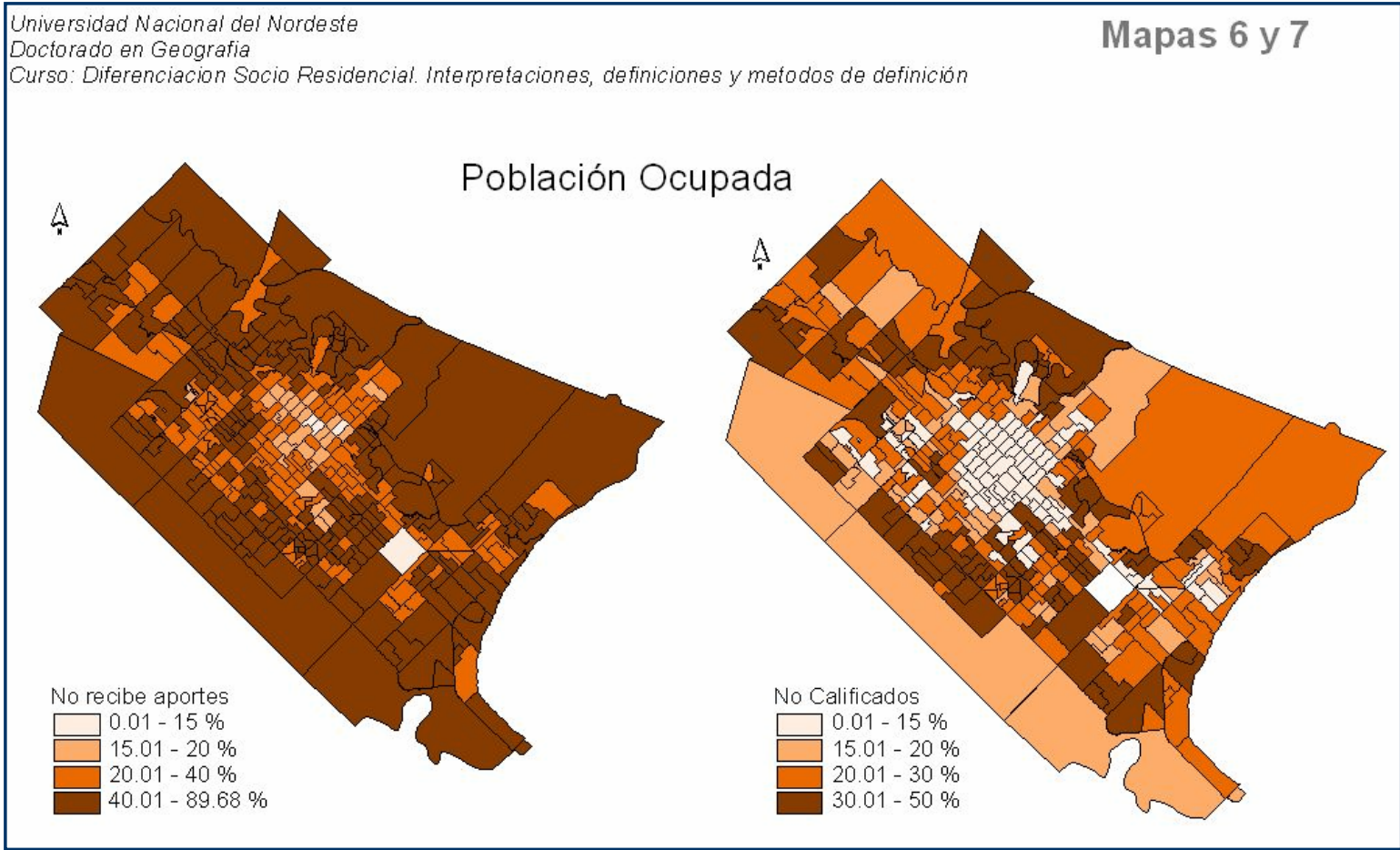

Los mapas que nos muestran la distribución espacial de estos cuatro elementos representados, dejan ver una correspondencia inversamente proporcional; por un lado, los espacios urbanos que presentan una mayor proporción de población con estudios universitarios completo y con asistencia a establecimientos educativos privados, corresponden a áreas urbanas con bajas proporciones de población ocupada no calificada o que no reciben aportes de ningún tipo. De acuerdo con esto podríamos inferir que la población asentada en los radios periféricos de la ciudad presentaría mayores inconvenientes para acceder a los estudios universitarios, hecho que podría generar mayores dificultades para insertarse en el mercado formal de trabajo.

$\mathrm{Si}$ a las distribuciones representadas agregamos otro indicador que refleja las condiciones socioeconómicas de la población, como lo es el tipo de Vivienda, podemos tener un elemento más para comprender el modo en que se da la diferenciación espacial en el espacio de estudio.

El mapa que muestra la distribución espacial de las viviendas tipos A y Departamentos permite observar una disposición en coronas o anillos determinados por altos valores -mayores al 82 $\%$ - que degradan desde los radios céntricos hacia la periferia, algunas veces expandidos linealmente siguiendo las principales avenidas, además de algunos sectores en formas de islas distribuidos en el conglomerado.

Publicado en formato digital: Mgtr. Vilma Lilian Falcón. DIFERENCIACION SOCIO RESIDENCIAL, UNA APLICACIÓN PRÁCTICA AL ESPACIO URBANO DEL GRAN RESISTENCIA. Revista Geográfica Digital. IGUNNE. Facultad de Humanidades. UNNE. Año 8. No 15. Enero - Junio 2011. ISSN 1668-5180 Resistencia, Chaco. En: http://hum.unne.edu.ar/revistas/geoweb/default.htm 


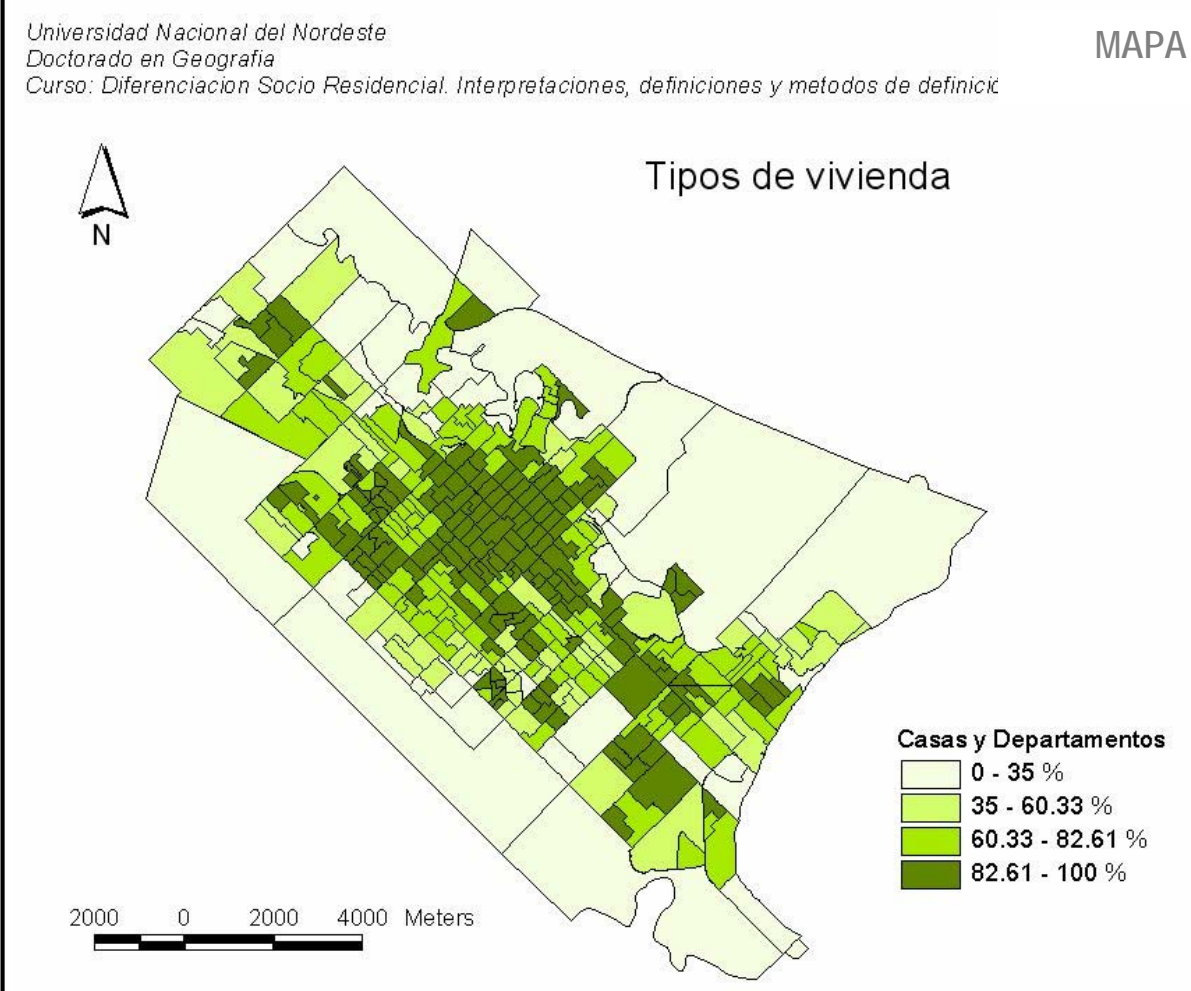

En el primer caso se trata del núcleo ya consolidado de la ciudad, el que se presenta con un mayor grado de continuidad y homogeneidad espacial en lo que hace a las características edilicias ${ }^{(6)}$ de este tipo de viviendas analizadas.

El segundo caso está conformado fundamentalmente por núcleos aislados que corresponden a grandes complejos habitacionales construido por el Estado en las décadas de los '80 y '90 y, en menor medida, a casos aislados de baja densidad poblacional pero con mayor Nivel Socioeconómico; todos ello insertos en un gran área de transición hacia la periferia y cuya característica es, precisamente, la heterogeneidad en cuanto a los tipos de viviendas

El tercer dominio conformado por las áreas periféricas donde el tipo de vivienda que se representa adquiere los menores valores (inferior al 35\%), vuelve a destacarse como un área homogénea; se trata de un espacio que bordea a las tres localidades del Gran Resistencia y que ya, en la época del relevamiento censal de 2001, comenzaba a delinear un esquema diferenciado entre la zona norte y sur ${ }^{(7)}$

Si tenemos en cuenta que este indicador refleja de un modo muy aproximado el nivel socioeconómico de la población, esta distribución viene a fortalecer algunas de las ideas expresadas al inicio de este trabajo, en el sentido de que nos permite afirmar que existe, en el Gran Resistencia, áreas periféricas con claros indicios de segregación urbana y por ende, de diferenciación socioespacial.

En síntesis, las distribuciones de las variables representadas en este segundo grupo de mapas, conservan de algún modo las formas ya observadas en el grupo anterior, pero al sumar otras variables la complejidad es cada vez mayor. Este hecho hace que encontremos una variante en la asociación con los modelos teóricos de ciudad que hemos señalado en el apartado anterior. En este caso, podemos señalar que existen -en la forma espacial que adquieren las variables socioeconómicas representadas-, elementos que podrían ligarse al modelo diseñado por Griffin y Ford $(1980)^{(8)}$ para las ciudades latinoamericanas pero destacando la combinación con el modelo sectorial de Hoytt. En el primer caso, porque se observa una disposición diferenciada de tres áreas que van desde el centro hacia la periferia y en el segundo, por una extensión sectorizada de las distribuciones, siguiendo las formas lineales de las principales avenidas del conglomerado. 
Revista Geográfica Digital. IGUNNE. Facultad de Humanidades. UNNE. Año 8. № 15. Enero - Junio 2011. ISSN 1668-5180 Resistencia, Chaco

\section{5.- Hacia la búsqueda de un modelo}

El análisis planteado en el punto anterior viene a confirmar que el Gran Resistencia se presenta como un espacio heterogéneo, con una clara diferenciación socio residencial de la población, diferencia que encuentra su mayor explicación en el comportamiento de las variables socieconómicas, fundamentalmente las que tienen que ver con el grupo etario de la población y la posesión de viviendas tipo A y Departamentos, tal como nos muestran los mapas correspondientes.

En lo que respecta a la distribución espacial se observa claramente un área central caracterizada por las mejores condiciones en el nivel de vida de la población y otras áreas sucesivas en las cuales dichas condiciones se van degradando hasta conformar un espacio homogéneo periférico que se destaca por las situaciones más críticas desde el punto de vista socioeconómico. En medio de estas áreas se reconocen algunas islas o sectores, algunas veces respondiendo a la presencia de importantes avenidas que comunican las 4 localidades que conforman el conglomerado y otras veces a las particularidades propias de los asentamientos en barrios planificados.

Si bien esto nos remite a los primeros modelos estudiados como los de los "anillos concéntricos" de Burguess o "de los sectores" de Hoytt, que hemos comentado en el análisis de la cartografía temática, y que consideramos válidos como esquema para nuestra ciudad, nos parece más apropiado enfocarnos en aquellos que trataron de modelizar a las ciudades latinoamericanas, aunque no nos alejaremos de aquella primera idea dado que los autores que proponemos -como tantos otros- se basan en esos esquemas tradicionales.

Por un lado, el modelo que proponen Griffin y Ford (1980 y 1996) para las ciudades latinoamericanas se ajustaría a la forma que asumen las distribuciones en el espacio urbano del Gran Resistencia pero destacando la combinación con el modelo sectorial de Hoytt (1939). Como ya lo señaláramos, en el primer caso, porque se observa una disposición diferenciada de áreas sucesivas que van desde el centro hacia la periferia y, en el segundo, por una extensión sectorizada de las distribuciones, siguiendo las formas lineales de las principales avenidas del conglomerado.

En la ciudad de Resistencia los anillos sucesivos se encuadran de un modo muy cercano a la que describe el modelo de Griffin y Ford ${ }^{(9)}$, en el sentido que se parte de un área central o CBD caracterizado como un espacio en el que se concentra la actividad comercial y financiera; en ese orden, dada la función comercial de nuestra ciudad. Este centro abarca apenas un radio de 5 cuadras alrededor de la plaza central. Inmediatamente después aparece lo que podríamos llamar la "zona de madurez" o al que también llamamos "macro-centro" al que sumamos otras 5 cuadras más y que quedarían enmarcadas dentro de las principales avenidas; esta zona se caracteriza por la presencia de viviendas de muy buena calidad, donde se disponen de todos los servicios, agua, luz, cloacas y fundamentalmente el pavimento que es el elemento de la infraestructura urbana que marca una diferencia sustancial en los distintos espacios de la ciudad.

El anillo que los autores llaman "de acrecimiento in situ" se da en nuestra ciudad como el espacio intermedio o de transición hacia la periferia, con la particularidad que aquí se entremezclan los tipos de viviendas, el nivel socioeconómico de la población y la infraestructura queda reducida a dos elementos: agua y luz y, en forma mas sectorizada la red de cloacas y pavimentos asociadas más bien a las construcciones de viviendas planificadas. Es en este anillo que las distribuciones representadas en los mapas aparecen como menos homogéneas, precisamente porque alternan allí distintas situaciones que se reflejan en las condiciones de vida o socioeconómicas de la población, interpuestas a veces por núcleos o sectores que presentan características de las dos áreas incluidas en el macro-centro (mejores condiciones de vivienda, población con estudios universitarios, mejor cualificación profesional, etc.etc.).

Por último, la zona periférica, es el lugar de residencia de una población más carenciada y donde los indicadores se presentan como más desfavorables desde el punto de vista de las condiciones de vida; es también la que cuenta con escasa infraestructura en la red de cloacas y pavimento.

Por otra parte, si consideramos el modelo teórico de las ciudades latinoamericanas que presentan Borsford y otros $(2005)^{(10)}$, diremos que la asociación del esquema con nuestra ciudad en estudio puede darse, pero en la etapa que los autores denominan como "la ciudad polarizada" (19501990). Esta consideración se fundamenta, en primer lugar, a que el Gran Resistencia no es la gran metrópolis que observan los autores como ejemplos en su estudio de los modelos, y por otro lado, a que Resistencia, hacia el año $2001^{(11)}$ aún presentaba todas las características de un centro que

Publicado en formato digital: Mgtr. Vilma Lilian Falcón. DIFERENCIACION SOCIO RESIDENCIAL, UNA APLICACIÓN PRÁCTICA AL ESPACIO URBANO DEL GRAN RESISTENCIA. Revista Geográfica Digital. IGUNNE. Facultad de Humanidades. UNNE. Año 8. No 15. Enero - Junio 2011. ISSN 1668-5180 Resistencia, Chaco. En: http://hum.unne.edu.ar/revistas/geoweb/default.htm 
crecía hacia la periferia a partir de asentamientos particulares de una población con medio a bajo poder adquisitivo y que encontraba en zonas alejadas del centro terrenos no muy costosos. A ello se sumaba una reactivación en la construcción de viviendas populares por parte del gobierno que utilizó grandes espacios más allá de un radio de por lo menos 30 cuadras desde el casco céntrico, manteniéndose todavía un esquema simple de crecimiento. Vale decir que, la complejidad que encierra el modelo de la etapa "ciudad fragmentada" que consideran los autores, no se manifestaba aún y recién comienza a ser visible en nuestra ciudad en años posteriores al 2001 y por lo tanto esas particularidades quedan fuera de nuestro análisis.

En este sentido, el hecho de habernos aproximado al conocimiento teórico de los modelos de las ciudades propuestos a través de la historia, nos permite afirmar que si bien la ciudad de Resistencia, hacia el año 2001, presenta un esquema de configuración que se asocia con los modelos descritos en párrafos anteriores, en la actualidad estamos observando una mutación hacia otro modelo o esquema, dada la aparición de nuevos elementos que tienen que ver con la infraestructura urbana, tales como la consolidación de redes viales, de aguas y cloacas en espacios alejados del macrocentro, y nuevos asentamientos antagónicos en la periferia: por un lado, espacios con viviendas de alta calidad en el sector Noreste de la ciudad y por otro lado, espacios con asentamientos de viviendas muy precarias en el sector sur-sureste. Por otra parte, en los últimos 5 años se sumaron las construcciones de edificios en altura en diversos puntos del macro-centro. Todo ello refuerza nuestra idea de que los modelos de ciudades son válidos, más que para describir su asociación con otros en un momento dado, para interpretar su posible evolución hacia otros esquemas de modo tal que se puedan prever distintas situaciones de cambios y planificar acertadamente un modelo de ciudad que sea útil a toda la sociedad.

\section{NOTAS}

(1) El curso de postgrado se desarrolló en el marco de actividades propuestas por el Doctorado en Geografía que se dicta en la Facultad de Humanidades de la Universidad Nacional del Nordeste, en el año 2009.-.

(2) Señala el autor que los diferentes modelos se distinguen según sus dimensiones temporales y espaciales. Dichos modelos revelan el estado actual o el desarrollo continuo, así como también las dimensiones de latitud y longitud (plano) o la tercera dimensión (perfil). El esquema que presenta en el trabajo citado, responde a esquemas estructurales generalizados de la ciudad en América Latina, situados siempre al final de importantes fases de la urbanización, como son la época colonial (1820), la primera fase de urbanización influida fuertemente por la inmigración europea (1920), la segunda fase de urbanización marcada por el éxodo rural y la migración interna (1970) y la ciudad contemporánea (2000). En estas etapas, la ciudad cambió desde un cuerpo muy compacto a un perímetro sectorial, desde un organismo polarizado a una ciudad fragmentada.

(3) Sabatini, Francisco (2003) La segregación social del espacio en las ciudades de América Latina. Documentos del Instituto de Estudios Urbanos y Territoriales. Serie Azul № 35. Julio, 2003.

(4) Tomamos lo que señala Buzai (2003: 64) en el sentido que el modelo propuesto por Burguess (1925) "se presenta como una construcción ideal de las características de expansión a partir del centro.....en este sentido, cada "anillo" sería producto de una etapa de expansión y estabilización de determinados usos del suelo".

(5) Este autor amplía el modelo de Burguess propuesto en 1925 y considera que el patrón general de renta del suelo no se distribuye espacialmente al azar, sino que presenta un patrón encontrado en todas las ciudades que tiende a acomodarse en forma de sectores. Estos sectores de uso del suelo se encuentran ligados principalmente a los mayores ejes de transporte, aunque también intervienen otros elementos como la topografía, aspectos paisajísticos entre otros.

(6) El Instituto Nacional de Estadística y Censos de la Argentina no define explícitamente las características de las Viviendas Tipo $A$, aunque señala que "todos los casos que no presentan características de tipo B, se considera como A. Las de tipo B se define como: la que presenta al menos una de las siguientes condiciones: tiene piso de tierra o ladrillo suelto $u$ otro material (no tiene piso de cerámica, baldosa, mosaico, mármol, madera, alfombra, cemento o ladrillo fijo); o no tiene provisión de agua por cañería dentro de la vivienda o no dispone de inodoro con descarga de agua.

(7) En la actualidad el esquema al que hacemos referencia continua acentuándose ya que los terrenos de la zona norte fueron adquiriendo en forma progresiva mayores valores, por lo tanto quienes pueden acceder a ellos tienen también el poder adquisitivo como para construir casas con las

Publicado en formato digital: Mgtr. Vilma Lilian Falcón. DIFERENCIACION SOCIO RESIDENCIAL, UNA APLICACIÓN PRÁCTICA AL ESPACIO URBANO DEL GRAN RESISTENCIA. Revista Geográfica Digital. IGUNNE. Facultad de Humanidades. UNNE. Año 8. No 15. Enero - Junio 2011. ISSN 1668-5180 Resistencia, Chaco. En: http://hum.unne.edu.ar/revistas/geoweb/default.htm 
condiciones óptimas en cuanto a materiales y diseño. Por su parte, precisamente desde el año 2001, la zona sur -especialmente la de Resistencia- fue ocupada por numerosos asentamientos con un alto grado de precariedad en la construcción de las viviendas.

(8) Griffin y Ford (1980) propusieron un modelo de ciudad que combina elementos tradicionales de la estructura urbana y elementos modernos que ya venían alterando la estructura de las ciudades latinoamericanas. La estructura de Griffin y Ford (1980) es sectorial y se basa en el modelo de Hoyt (1939). El anillo del medio es una zona intermedia, de transición entre el centro y las zonas residenciales donde se registran diferentes tipo de vivienda de diferentes calidades y tamaños. La zona exterior corresponde a los asentamientos periféricos que se presenta como una zona residencial, que carece de servicios y donde vive la gente de menores recursos.

Para Griffin y Ford, las ciudades latinoamericanas se desarrollan según aspectos culturales, económicos y sociales propios de cada país. (CACACE, Graciela. En: http://www.geogra.uah.es/inicio/web 11 confibsig/PONENCIAS/2-020-Cacace.pdf)

(9) Para la descripción de las característica de cada uno de los anillos nos basamos en el trabajo de Buzai, G. 2003:90, que resume de una manera muy clara las particularidades de cada caso, aún más detallada que la figura gráfica que presenta el esquema del modelo.

(10) Bhär, Jürgen y Borsford, Axel (2005). La ciudad Latinoamericana, la construcción de un modelo. Vigencias y perspectivas. En: ur(b)es. Año II, No 2, Lima, Noviembre de 2005- pp. 207-221

(11) Recordemos que estamos analizando un comportamiento basado en datos censales del año 2001.

\section{BIBLIOGRAFIA}

BHÄR, Jürgen y BORSFORD, Axel (2005). La ciudad Latinoamericana, la construcción de un modelo. Vigencias y perspectivas. En: ur(b)es. Año II, № 2, Lima, Noviembre de 2005- pp. 207221.

BORSDORF, Axel (2003) Cómo modelar el desarrollo y la dinámica de la ciudad

latinoamericana. EURE (Santiago) [online]. Vol.29, n.86 [citado 2009-10-06], pp. 37-49. Disponible en: <http://www.scielo.cl/scielo.php?script=sci_arttext\&pid=S0250-

$71612003008600002 \&$ Ing=es\&nrm=iso>. ISSN doi: 10.4067/S0250-71612003008600002.

BUZAI, Gustavo (2003). Mapas Sociales Urbanos. Lugar Editorial S.A., Buenos Aires.

BUZAI, Gustavo y BAXENDALE, Claudia (2006). Análisis Espacial con Sistemas de Información Geográfica. Lugar Editorial S.A.- GEPAMA. Buenos Aires.

BRUNIARD, Enrique (1995).Las regiones homogéneas en Geografía Humana. Guia de Trabajos Prácticos. En: NORDESTE, 2da. Época. № 1. Serie Docencia. Facultad de Humanidades, UNNE. Chaco.

JANOSCHKA, Michael (2000) El nuevo modelo de la ciudad latinoamericana: fragmentación y privatización. EURE (Santiago) [online]. vol.28, n.85 [citado 2009-10-19], pp. 11-20 . Disponible en: $<$ http://www.scielo.cl/scielo.php?script=sci_arttext\&pid=S0250-

$71612002008500002 \& \operatorname{lng}=e s \& n r m=i s o>$. ISSN . doi: $10.4067 / S 0250-71612002008500002$.

JUAREZ MARTINEZ, María L. (2006). Segregación urbana y sus implicaciones en las ciudades. Una aproximación teórica. En: Palapa, Año $1 \mathrm{~N}^{\circ} 002$. Universidad de Colima, México.

NATERAS RIVAS, Juan y Gómez, Nestor J.. (2007). Diferenciación socio residencial en el aglomerado del Gran Santa Fe (Argentina) a comienzos del siglo XXI. Revista Universitaria de Geografia. Vol. 6 NN$^{\circ} 1$.

RODRÍGUEZ VIGNOLI, Jorge (2001). Segregación residencial socioeconómica: ¿qué es?, ¿cómo se mide?, ¿qué está pasando?, ¿importa?. Serie Población y Desarrollo. № 16. CEPALECLAC. Chile, Agosto 2001.-

SABATINI, Francisco (2003) La segregación social del espacio en las ciudades de América Latina. Documentos del Instituto de Estudios Urbanos y Territoriales. Serie Azul № 35. Julio, 2003 VALDEZ, Estela. Fragmentación y segregación urbana. Aportes teóricos para el análisis de casos en la ciudad de Córdoba. En: http://www.ffyh.unc.edu.ar/alfilo/alfilo-18/pdf/valdes.pdf 